\title{
The lattice of congruence lattices of algebras on a finite set
}

\author{
Danica Jakubíková-Studenovská* \\ Institute of Mathematics \\ P.J. Šafárik University \\ Košice (Slovakia) \\ Sándor Radeleczki ** \\ Institute of Mathematics \\ University of Miskolc (Hungary) \\ Dedicated to E. Tamás Schmidt \\ (version February 23, 2017)
}

\begin{abstract}
The congruence lattices of all algebras defined on a fixed finite set $A$ ordered by inclusion form a finite atomistic lattice $\mathcal{E}$. We describe the atoms and coatoms. Each meet-irreducible element of $\mathcal{E}$ being determined by a single unary mapping on $A$, we characterize completely those which are determined by a permutation or by an acyclic mapping on the set $A$. Using these characterisations we deduce several properties of the lattice $\mathcal{E}$; in particular, we prove that $\mathcal{E}$ is tolerance-simple whenever $|A| \geq 4$.
\end{abstract}

\section{Introduction}

In 1963 G. GrÄtzer and E.T. SChMidt proved that every algebraic lattice is isomorphic to the congruence lattice of some algebra ([GräS63]). Since the algebras constructed by them were infinite, the result immediately raised the question: Does every finite lattice occur as the congruence lattice of a finite algebra? The problem remained open till today, and it is usually referred as the finite lattice representation problem. It is an abstract representation problem because it asks for a solution up to isomorphism. The concrete version is the more involved question: Given a sublattice $E$ of the the partition lattice $\operatorname{Eq}(A)$ of all equivalence relations on a set $A$, does there exist an algebra on the same base set $A$, such that $E$ equals the congruence lattice of this algebra (in [Wer76] such lattices $E$ are characterized by closure properties).

\footnotetext{
* supported by Slovak VEGA grant 1/0063/14

** This research started as part of the TAMOP-4.2.1.B-10/2/KONV-2010-0001 project, supported by the European Union, co-financed by the European Social Fund 113/173/0-2.
} 
The subject of the present paper is related to the finite representation problem in its concrete version. For a fixed finite set $A$ we consider all possible congruence lattices of algebras with base set $A$. These congruence lattices (ordered by inclusion) form itself a lattice $\mathcal{E}$ and we are going to investigate this lattice. An important tool is our knowledge about the lattice $\mathcal{L}$ of all quasiorder lattices of algebras defined on the set $A$ described in [JakPR16] (using some techniques developed previously in the papers [JakPR11] and [JakPR13]). These two lattices are strongly interrelated: there is a residual mapping from $\mathcal{L}$ to $\mathcal{E}$. Therefore, in Section 3, we investigate on abstract level, how lattice properties (which are relevant for us) behave under residual mappings (for instance, the coatoms of $\mathcal{E}$ directly can be obtained from the coatoms of $\mathcal{L}$, see 3.1(iv)).

Based on preliminary results from Section 2 and the results of [JakPR16] and Section 3, we describe the atoms ( $\vee$-irreducible elements), coatoms (Section 4) and further $\wedge$-irreducible elements (Sections 5 and 6 ) of the lattice $\mathcal{E}$. Finally, in Section 7, we investigate several lattice theoretic properties of $\mathcal{E}$, e.g., it is tolerance simple, but has no properties related with modularity.

\section{Preliminaries}

Throughout the paper we fix a base set $A$ (if not stated otherwise, $A$ is assumed to be finite). Further, let $\mathbb{N}:=\{0,1,2, \ldots\}$ and $\mathbb{N}_{+}:=\mathbb{N} \backslash\{0\}$. For a mapping $f: A \rightarrow A$, we write $f a$ for the image of an element $a \in A$, and $f^{n}(n \in \mathbb{N})$ denotes the $n$-fold composition of $f$ (by convention, $f^{0}$ is the identity mapping $\left.\operatorname{id}_{A}\right)$.

2.1 Definitions. Let $\operatorname{Eq}(A)$ and $\operatorname{Quord}(A)$ denote the set of all equivalence relations (reflexive, symmetric and transitive) and quasiorders (reflexive and transitive relations), respectively, on a set $A$. The least and the greatest quasiorders (which are in fact equivalences) are $\Delta:=\{(x, x) \mid x \in A\}$ and $\nabla:=A \times A$. A unary mapping $f: A \rightarrow A$ preserves a quasiorder $q \in \operatorname{Quord}(A)$ (in particular, an equivalence $q=\varkappa \in \mathrm{Eq}(A))$, notation $f \triangleright q$, if

$$
\forall x, y \in A:(x, y) \in q \Longrightarrow(f x, f y) \in q .
$$

This fact is also expressed by the following notions and notation: $f$ is an endomorphism of $q(f \in \operatorname{End} q), q$ is invariant for or compatible with $f$, or $q$ is a quasiorder of $(A, f)(q \in \operatorname{Quord}(A, f))$, or $\varkappa$ is a congruence of $(A, f)$ $(\varkappa \in \operatorname{Con}(A, f))$.

The identity $\operatorname{id}_{A}: A \rightarrow A: x \mapsto x$ as well as all constant mappings $A \rightarrow A: x \mapsto$ $a$ are called trivial because just they preserve every quasiorder $q \in \operatorname{Quord}(A)$. For a unary algebra $(A, F), F \subseteq A^{A}$, let $\operatorname{Con}(A, F)$ and $\operatorname{Quord}(A, F)$ be its congruence and quasiorder lattice, respectively, i.e. the lattice of all equivalences or quasiorders that are compatible with each $f \in F$. Moreover, let

$$
\mathcal{E}:=\left\{\operatorname{Con}(A, F) \mid F \subseteq A^{A}\right\} \text { and } \mathcal{L}:=\left\{\operatorname{Quord}(A, F) \mid F \subseteq A^{A}\right\}
$$


denote the lattice of all such congruence lattices and quasiorder lattices, respectively, on $A$, ordered by inclusion. Instead of $\operatorname{Quord}(A, F)$ and $\operatorname{Con}(A, F)$ we sometimes write simply Quord $F$ and Con $F$. Since congruences of an algebra are characterized by the unary polynomial functions of the algebra, the lattice $\mathcal{E}$ is in fact the lattice of all congruence lattices of arbitrary (not necessarily unary) algebras on the set $A$ (the same holds for quasiorders and $\mathcal{L}$ ).

2.2 Remarks. The relation $\triangleright$ induces (via the operators Con and End) a Galois connection between unary mappings and equivalence relations on $A$. The Galois closures are just the elements (congruence lattices) $\operatorname{Con}(A, F) \in \mathcal{E}$ and monoids of the form End $Q$ (for some set $Q \subseteq \mathrm{Eq}(A)$ ), in particular, we have

$$
E \in \mathcal{E} \Longleftrightarrow E=\operatorname{Con}(A, \text { End } E) \text { (i.e., } E \text { is Galois closed). }
$$

The meet in $\mathcal{E}$ is the intersection while the join of elements $E_{i} \in \mathcal{E}(i \in I)$ is given by $\bigvee_{i \in I} E_{i}=$ Con End $\bigcup_{i \in I} E_{i}$.

Clearly, $F \subseteq F^{\prime}$ implies $\operatorname{Con}\left(A, F^{\prime}\right) \subseteq \operatorname{Con}(A, F)$. Thus $\wedge$-irreducibles in $\mathcal{E}$ must be of the form $\operatorname{Con}(A, f)$ for a single function $f$ because $\operatorname{Con}(A, F)$ is the intersection of all $\operatorname{Con}(A, f)$ with $f \in F$. Analogously, $\vee$-irreducible (in case of infinite $A$, completely $\vee$-irreducible) elements of $\mathcal{E}$ must be of the form $E_{\varkappa}:=$ Con End $\varkappa$ for a single equivalence relation $\varkappa \in \operatorname{Eq}(A) \backslash\{\Delta, \nabla\}$, because, for $E \in \mathcal{E}$, End $E$ is the intersection of all End $\varkappa$ and thus Con End $E=E$ is the join (in $\mathcal{E}$ ) of all Con End $\varkappa$ with $\varkappa \in E$.

2.3 Notation. For $\varkappa \in \operatorname{Eq}(A)$ consider the corresponding partition $A / \varkappa$ into equivalence classes. If $C_{1}=\left\{a_{1}, a_{2}, \ldots\right\}, C_{2}=\left\{b_{1}, b_{2}, \ldots\right\}, \ldots, C_{m}=$ $\left\{c_{1}, c_{2}, \ldots\right\}$ are the equivalence classes of $\varkappa$ with at least two elements, then we use the notation

$$
\begin{aligned}
\varkappa & =\left[a_{1}, a_{2}, \ldots\right]\left[b_{1}, b_{2}, \ldots\right] \ldots\left[c_{1}, c_{2}, \ldots\right] \text { or } \\
\varkappa & =\left[C_{1}\right]\left[C_{2}\right] \ldots\left[C_{m}\right] .
\end{aligned}
$$

All other elements which do not appear in the list form one-element equivalence classes.

2.4 Monounary algebras. Here we introduce some special notions for monounary algebras; for a more general view to monounary algebras we refer to [JakP09].

Let $(A, f)$ be a finite monounary algebra. Let $Z_{f}(x):=\left\{f^{i} x \mid i \in \mathbb{N}\right\}$ be the subalgebra of $(A, f)$ generated by an element $x \in A$. Obviously, we have $a \in Z_{f}(x) \Longleftrightarrow Z_{f}(a) \subseteq Z_{f}(x)$. We write $B \leq(A, f)$ if $B$ is (the carrier set of) a subalgebra of $(A, f)$.

Considering the graph $f^{\bullet}:=\left\{(a, b) \in A^{2} \mid b=f a\right\}$ of $f$, one can use a graph theoretic terminology. For $a \in A$, let $K_{f}(a)$ denote the connected component of $f^{\bullet}$ to which $a$ belongs (note that two vertices $x, y \in A$ are connected w.r.t. $f$, iff there exist $i, j \in \mathbb{N}$ with $f^{i} x=f^{j} y$ ). A component $K$ of $f$ is called nontrivial if it contains at least two elements (thus a trivial component is just a fixed point). 
For a monounary algebra $(A, f)$, the least quasiorder and congruence, resp., containing a pair $(x, y) \in A^{2}$ is denoted by $\alpha_{f}(x, y)$ and $\theta_{f}(x, y)$ (principal congruence), resp., and we have

$$
\begin{aligned}
\alpha_{f}(x, y) & =\Delta \cup\left\{\left(f^{i} x, f^{i} y\right) \mid i \in \mathbb{N}\right\}^{\text {tra }}, \\
\theta_{f}(x, y) & =\Delta \cup\left\{\left(f^{i} x, f^{i} y\right) \mid i \in \mathbb{N}\right\}^{\text {sym tra }} .
\end{aligned}
$$

Here $\Psi^{\text {sym }}=\Psi \cup \Psi^{-1}$ denotes the symmetric closure and $\Psi^{\text {tra }}$ the transitive closure of a binary relation $\Psi \subseteq A \times A$.

We now collect some properties for functions $f, g \in A^{A}$ with $\operatorname{Con}(A, f) \subseteq$ $\operatorname{Con}(A, g)$.

2.5 Lemma. Let $f, g \in A^{A}$ be nontrivial and $\operatorname{Con}(A, f) \subseteq \operatorname{Con}(A, g)$. Then we have

(i) $\forall x, y \in A:(x, y) \in \varkappa \in \operatorname{Con}(A, f) \Longrightarrow(g x, g y) \in \varkappa$, in particular we have $(g x, g y) \in \theta_{f}(x, y)$ and $\theta_{g}(x, y) \subseteq \theta_{f}(x, y)$.

(ii) Let $B$ be a subalgebra of $(A, f)$. Then either $B$ is also a subalgebra of $(A, g)$ or $g$ is constant on $B$, where the constant does not belong to $B$.

Proof. (i) is clear since $f \triangleright \varkappa$ implies $g \triangleright \varkappa$ what follows from the assumption $\operatorname{Con}(A, f) \subseteq \operatorname{Con}(A, g)$.

(ii): For a subalgebra $B, \varepsilon_{B}:=\Delta \cup B \times B$ belongs to $\operatorname{Con}(A, f)$. Let $x \in B$. If $g$ is not constant on $B$, then there exists $y \in B$ such that $g x \neq g y$. Because $(x, y) \in \varepsilon_{B}$, by (i) we have $(g x, g y) \in \varepsilon_{B} \backslash \Delta$, in particular $g x \in B$. Thus $B$ is closed under $g$. If $g$ is constant on $B$ and $B$ is not a subalgebra of $(A, g)$, then the constant cannot be an element of $B$.

2.6 Remark. The property in 2.5(i) completely characterizes the containment of the congruence lattices. We have for $f, g \in A^{A}$ :

$$
\operatorname{Con}(A, f) \subseteq \operatorname{Con}(A, g) \Longleftrightarrow \forall x, y \in A:(g x, g y) \in \theta_{f}(x, y) .
$$

In preparation of the next proposition we need the following lemma.

2.7 Lemma. Let $f$ be a permutation of prime power order $p^{m}$ with at least two cycles of length $p^{m}$. Then End $\operatorname{Con}(A, f)=$ End $\operatorname{Quord}(A, f)$.

Proof. The inclusion " $\supseteq$ " is always true. To show " $\subseteq$ ", let $h \notin \operatorname{End} \operatorname{Quord}(A, f)$. Thus there exist $\varrho \in \operatorname{Quord}(A, f)$ with $h \not \varrho$ and therefore some principal quasiorder $\alpha_{f}(x, y)$ which is not preserved by $h$ for some $(x, y) \in \varrho$. We must show $h \notin$ End $\operatorname{Con}(A, f)$. Assume on the contrary that $h \in \operatorname{End} \operatorname{Con}(A, f)$ or, equivalently, $\operatorname{Con}(A, f) \subseteq \operatorname{Con}(A, h)$. W.l.o.g. we can assume $(h x, h y) \notin \alpha_{f}(x, y)$ (because there must exist $(u, v) \in \alpha_{f}(x, y)$ with $(h u, h v) \notin \alpha_{f}(u, v) \subseteq \alpha_{f}(x, y)$, one can use $(u, v)$ instead of $(x, y))$. 
If $x, y$ belong to the same cycle of the permutation $f$, then $\alpha_{f}(x, y)=\theta_{f}(x, y)$ (cf. [Jak09, Lemma 3.1]) and we have $h \not \theta_{f}(x, y)$, a contradiction. Thus we may assume $x \in C_{1}, y \in C_{2}$ where $C_{1}, C_{2}$ are different cycles of $f$ of length $p^{k_{1}}$ and $p^{k_{2}}$, resp. Moreover, w.l.o.g. assume $k_{1} \geq k_{2}$. Then we have $\alpha:=$ $\alpha_{f}(x, y)=\Delta \cup\left\{\left(f^{i} x, f^{i} y\right) \mid 0 \leq i \leq p^{k_{1}}-1\right\}$ and $\theta:=\theta_{f}(x, y)=\alpha \cup \alpha^{-1} \cup \beta$ where $\beta:=\left\{\left(f^{i} x, f^{j} x\right) \mid i, j \in\left\{0,1, \ldots, p^{k_{1}}-1\right\}, j \equiv i\left(\bmod p^{k_{2}}\right)\right\}$; note that $\beta \subseteq C_{1} \times C_{1}$.

We distinguish the following cases (recall $h \triangleright \theta$ and hence $(h x, h y) \in \theta \backslash \alpha)$ :

Case 1: $(h x, h y) \in \alpha^{-1}$, i.e., $(h x, h y)=\left(f^{i} y, f^{i} x\right) \in C_{2} \times C_{1}$ for some $i$. By Lemma 2.5(ii), $h$ is constant $f^{i} x$ on $A \backslash C_{1} \leq(A, f)$ and constant $f^{i} y$ on $A \backslash C_{2} \leq(A, f)$. If there exists $c \in A \backslash\left(C_{1} \cup C_{2}\right)$, then $f^{i} x=h c=f^{i} y$, a contradiction. Thus $A=C_{1} \cup C_{2}$, i.e., $C_{1}$ and $C_{2}$ must be two cycles of length $p^{m}$. But then $h$ does not preserve $\theta_{f}(x, f y)$ since $(h x, h y)=\left(f^{i} y, f^{i} x\right) \notin$ $\theta_{f}(x, f y)=[x, f y]\left[f x, f^{2} y\right] \ldots\left[f^{i} x, f^{i+1} y\right] \ldots$, a contradiction.

Case 2: $(h x, h y) \in \beta$, hence $(h x, h y) \in C_{1} \times C_{1}$. In particular we have $\left|C_{1}\right|>1$, i.e., $k_{1} \geq 1$. Further, by 2.5 (ii), $h$ is constant $h y \in C_{1}$ on $A \backslash C_{1} \leq(A, f)$.

If $k_{1}=k_{2}=m$, then $(h x, h y) \in\left(C_{1} \times C_{1}\right) \cap \theta_{f}(x, y) \subseteq \Delta$, a contradiction.

If $k_{2}<m$, then there exists a cycle $C$ of length $p^{m}$ which is distinct from $C_{1}$. Let $x_{0} \in C$. Then each block of $\theta_{f}\left(x_{0}, x\right)$ contains exactly one element of $C_{1}$, hence $\left(h x_{0}, h x\right)=(h y, h x) \notin \theta_{f}\left(x_{0}, x\right)$, a contradiction to $h \triangleright \theta_{f}\left(x_{0}, x\right) \in \operatorname{Con}(A, f)$.

Thus the assumption $h \in$ End $\operatorname{Con}(A, f)$ must fail, i.e., $h \notin$ End $\operatorname{Con}(A, f)$.

2.8 Proposition. Let $f, g \in A^{A}$ be nontrivial such that $\operatorname{Con}(A, f) \subseteq \operatorname{Con}(A, g)$ and let $f$ be a permutation of prime power order $p^{m}$ with at least two cycles of length $p^{m}$. Then there exists $k \in\left\{1, \ldots, p^{m}-1\right\}$ such that $g=f^{k}$.

Proof. $\operatorname{Con}(A, f) \subseteq \operatorname{Con}(A, g)$ is equivalent to $g \in \operatorname{End} \operatorname{Con}(A, f)$, thus $g \in$ End $\operatorname{Quord}(A, f)$ by Lemma 2.7, consequently $\operatorname{Quord}(A, f) \subseteq \operatorname{Quord}(A, g)$. From [JakPR16, Proposition 2.5(b)] we conclude $\exists k \in \mathbb{N}_{+}: g=f^{k}$. Clearly $k$ can be chosen less than $p^{m}$ since $f^{p^{m}}=\mathrm{id}_{A}$.

\section{Residual mappings and $\wedge$-irreducibles}

We shall strongly use results about the lattice $\mathcal{L}$ of quasiorder lattices for the investigation of the lattice $\mathcal{E}$ of congruence lattices. However, we want to seperate those connections which are of pure lattice theoretic nature (and which are from our point of view - of its own interest). This is done in this section. Based on the observation that $\Phi: \mathcal{L} \rightarrow \mathcal{E}: Q \mapsto Q \cap \operatorname{Eq}(A)$ is a residual mapping, we consider this case in a general setting.

Let $L$ and $E$ be arbitrary lattices which - for simplicity - here are assumed to be finite, the least and largest elements are denoted by $0_{L}, 0_{E}$ and $1_{L}, 1_{E}$. A mapping $\varphi: L \rightarrow E$ is called residual if it is a $\wedge$-homomorphism (and therefore also monoton with respect to the lattice orders) and $\varphi\left(1_{L}\right)=1_{E}$ (cf. e.g. [Jan94] or [JanR15]). 
The following proposition shows that then the $\wedge$-irreducible elements of $\mathcal{E}$, in particular coatoms, can be constructed from the $\wedge$-irreducible elements of $\mathcal{L}$.

3.1 Proposition. Let $\varphi: L \rightarrow E$ be a surjective residual mapping.

(i) Let $m \in E$ be a $\wedge$-irreducible element in $E$. Then $\varphi^{-1}(m) \neq \emptyset$ and each $q \in L$ which is maximal in $\varphi^{-1}(m)$ is $\wedge$-irreducible in $L$.

(ii) Let $m \in E$ and let each $q \in L$ with $\varphi(q)=m$ be $\wedge$-irreducible in $L$. Then $m$ is $\wedge$-irreducible in $E$.

(iii) Assume

$$
\varphi(x)=1_{E} \Longrightarrow x=1_{L} \text { for all } x \in L .
$$

Then for each coatom $m \in E$ there exists a coatom in $q \in L$ such that $\varphi(q)=m$.

(iv) Assume condition (†) above and

$$
\varphi(q) \leq \varphi\left(q^{\prime}\right) \Longrightarrow \varphi(q)=\varphi\left(q^{\prime}\right) \text { for all coatoms } q, q^{\prime} \in L .
$$

Then $\varphi(q)$ is a coatom in $E$ if $q$ is a coatom in L. Moreover, the set of all coatoms of $E$ is $\{\varphi(q) \mid q$ coatom in $L\}$.

Proof. (i): Let $m \in E$ be $\wedge$-irreducible. Then $\varphi^{-1}(m)=\left\{q^{\prime} \in L \mid \varphi\left(q^{\prime}\right)=m\right\}$ is nonempty because $\varphi$ is surjective. Let $q$ be maximal in $\varphi^{-1}(m)$. Then $q$ is the meet of $\wedge$-irreducible elements, say $q=q_{1} \wedge \ldots \wedge q_{s}$ with $\wedge$-irreducible $q_{i} \in L(i \in\{1, \ldots, s\})$. It follows $m=\varphi(q)=\varphi\left(q_{1}\right) \wedge \ldots \wedge \varphi\left(q_{s}\right)$. Because $m$ is $\wedge$-irreducible there exists $i \in\{1, \ldots, s\}$ such that $m=\varphi\left(q_{i}\right)$. Since $q \leq q_{i}$ and $q$ was chosen maximal with respect to $\varphi(q)=m$, we have $q=q_{i}$, i.e., it is $\wedge$-irreducible.

(ii): Let $m=m_{1} \wedge m_{2}$ for some $m_{1}, m_{2} \in E$. Since $\varphi$ is surjective, there exist $q_{i} \in L$ with $\varphi\left(q_{i}\right)=m_{i}, i \in\{1,2\}$. Let $q:=q_{1} \wedge q_{2}$. Then $\varphi(q)=\varphi\left(q_{1} \wedge q_{2}\right)=$ $\varphi\left(q_{1}\right) \wedge \varphi\left(q_{2}\right)=m_{1} \wedge m_{2}=m$ and $q$ must be $\wedge$-irreducible by assumption. Consequently, there is $i \in\{1,2\}$ with $q=q_{i}$, thus $m=\varphi(q)=\varphi\left(q_{i}\right)=m_{i}$, i.e., $m$ is $\wedge$-irreducible.

(iii): Since $L$ is finite, by (i) there exists a maximal $\wedge$-irreducible $q \in L$ with $\varphi(q)=m$. If $q$ were not a coatom then there would exist a $q^{\prime} \in L$ with $q<$ $q^{\prime}<1_{L}$. By the maximality property of $q$, we get $\varphi\left(q^{\prime}\right)>m$, thus $\varphi\left(q^{\prime}\right)=1_{E}$ (since $m$ is coatom) and by the assumption from (iii) we would get $q^{\prime}=1_{L}$, a contradiction.

(iv): Let $q \in L$ be a coatom. Then $\varphi(q) \neq 1_{E}$ because of $(\dagger)$. Thus there exists some coatom $m$ in $E$ with $\varphi(q) \leq m$. By (iii) there exists a coatom $q^{\prime}$ in $L$ such that $\varphi\left(q^{\prime}\right)=m$. Then $\varphi(q) \leq \varphi\left(q^{\prime}\right)$ and with $(\ddagger)$ we get that $\varphi(q)=\varphi\left(q^{\prime}\right)=m$ is a coatom in $E$. This together with (iii) shows that $\{\varphi(q) \mid q$ coatom in $L\}$ is the set of all coatoms of $E$. 
3.2 Remark. Concerning 3.1(i), since $L$ is finite, for any $q^{\prime} \in \varphi^{-1}(m)$ there exists a maximal (and therefore $\wedge$-irreducible) $q$ with $q^{\prime} \leq q \in \varphi^{-1}(m)$.

3.3 Application. There are many applications of residual mappings in various contexts, in particular in connection with the unique corresponding residuated mapping (establishing a "covariant Galois connection"). However, for this paper the only example which we need is the above mentioned residual mapping

$$
\Phi: \mathcal{L} \rightarrow \mathcal{E}: Q \mapsto Q \cap \operatorname{Eq}(A),
$$

$\left(\operatorname{recall} \mathcal{L}:=\left\{\operatorname{Quord}(A, F) \mid F \subseteq A^{A}\right\}\right.$ and $\mathcal{E}:=\left\{\operatorname{Con}(A, F) \mid F \subseteq A^{A}\right\}$ from 2.1). Clearly, $\Phi(\operatorname{Quord}(A, F))=\operatorname{Con}(A, F) \in \mathcal{E}$. The next Lemma shows that the assumptions $(\dagger)$ and $(\ddagger)$ in Proposition 3.1(iii),(iv) are satisfied for this example. Notice that $\operatorname{Eq}(A)$ and $\operatorname{Quord}(A)$ are the greatest elements of the lattices $\mathcal{E}$ and $\mathcal{L}$, respectively.

3.4 Lemma. (i) For $Q \in \mathcal{L}, \Phi(Q)=\operatorname{Eq}(A)$ implies $Q=\operatorname{Quord}(A)$.

(ii) For coatoms $Q, Q^{\prime}$ in $\mathcal{L}, \Phi(Q) \subseteq \Phi\left(Q^{\prime}\right)$ implies $\Phi(Q)=\Phi\left(Q^{\prime}\right)$.

Proof. (i): It is well-known that trivial functions ( $\mathrm{id}_{A}$ and the constants, say $C$ ) are the only mappings which preserves all equivalence relations. Thus we have $Q=$ Quord End $Q \supseteq$ Quord End $\Phi(Q)=$ Quord End $\operatorname{Eq}(A)=\operatorname{Quord}\left(\left\{\operatorname{id}_{A}\right\} \cup\right.$ $C)=\operatorname{Quord}(A)$.

(ii): This will follow immediately from Proposition 4.8 proved in Section 4.

Note that the coatoms of $\mathcal{E}$ and $\mathcal{L}$ are of the form $\operatorname{Con}(A, f)$ and $\operatorname{Quord}(A, f)$ for some specific $f$ (they are of type (I)-(III) as we shall see in Theorem 4.3). Thus from 3.1(iv) we immediately get:

3.5 Corollary. $\{\operatorname{Con}(A, f) \mid \operatorname{Quord}(A, f)$ is a coatom in $\mathcal{L}\}$ is the set of all coatoms of $\mathcal{E}$.

We close this section with two results which shall turn out to be useful later. For an element $C$ of a lattice $\mathcal{E}$ let $[C\rangle_{\mathcal{E}}:=\left\{C^{\prime} \in \mathcal{E} \mid C \leq C^{\prime}\right\}$ denote the principal filter generated by $C$.

3.6 Lemma. For $C \in \mathcal{E}$ and $Q:=$ Quord End $C$ we have $\Phi^{-1}\left([C\rangle_{\mathcal{E}}\right)=[Q\rangle_{\mathcal{L}}$.

Proof. Recall $C \in \mathcal{E} \Longleftrightarrow$ Con End $C=C$ and $Q^{\prime} \in \mathcal{L} \Longleftrightarrow$ Quord End $Q^{\prime}=$ $Q^{\prime}$. Now, if $Q^{\prime} \in[Q\rangle_{\mathcal{L}}$ then $\Phi\left(Q^{\prime}\right) \in[\Phi(Q)\rangle_{\mathcal{E}}=[C\rangle_{\mathcal{E}}$ since $\Phi$ is order preserving and $\Phi(Q)=\operatorname{Eq}(A) \cap$ Quord End $C=$ Con End $C=C$. Thus $Q^{\prime} \in$ $\Phi^{-1}\left([C\rangle_{\mathcal{E}}\right)$. Conversely, if $Q^{\prime} \in \Phi^{-1}\left([C\rangle_{\mathcal{E}}\right)$, then $C \subseteq \Phi\left(Q^{\prime}\right) \subseteq Q^{\prime}$ and we get $Q=$ Quord End $C \subseteq$ Quord End $Q^{\prime}=Q^{\prime}$, i.e., $Q^{\prime} \in[Q\rangle_{\mathcal{L}}$.

3.7 Corollary. For $F \subseteq A^{A}$ we have End $\operatorname{Quord}(A, F)=\operatorname{End} \operatorname{Con}(A, F)$ if and only if $\Phi^{-1}\left([\operatorname{Con}(A, F)\rangle_{\mathcal{E}}\right)=[\operatorname{Quord}(A, F)\rangle_{\mathcal{L}}$. 
Proof. Let $Q:=\operatorname{Quord}(A, F)$ and $C:=\operatorname{Con}(A, F)$. Then we have $C=\Phi(Q) \subseteq$ $Q$. If End $Q=$ End $C$ then $Q=$ Quord End $Q=$ Quord End $C$ and we get $\Phi^{-1}\left([C\rangle_{\mathcal{E}}\right)=[Q\rangle_{\mathcal{L}}$ from Lemma 3.6. Conversely, assume $\Phi^{-1}\left([C\rangle_{\mathcal{E}}\right)=[Q\rangle_{\mathcal{L}}$ and let $Q^{\prime}:=$ Quord End $C$. Then $\Phi\left(Q^{\prime}\right)=$ Con End $C=C$, thus $Q^{\prime} \in \Phi^{-1}\left([C\rangle_{\mathcal{E}}\right)$. Consequently, $Q^{\prime} \in[Q\rangle_{\mathcal{L}}$, i.e., $Q \subseteq Q^{\prime}=$ Quord End $C$, and we get End $Q \supseteq$ End Quord End $C \supseteq$ End $C \supseteq$ End $Q$ (the latter inclusion follow from $C \subseteq Q$ ), hence End $Q=$ End $C$.

\section{Atoms and coatoms of $\mathcal{E}$}

In this section we are going to describe the atoms ( $\vee$-irreducibles) and coatoms of $\mathcal{E}$. The case $|A|=2$ is trivial. Then $\mathcal{E}$ consists only of one lattice, namely $\operatorname{Con}\left(A, A^{A}\right)=\operatorname{Con}\left(A, \mathrm{id}_{A}\right)=\operatorname{Eq}(A)=\{\Delta, \nabla\}$. Therefore, in the following we assume always $|A| \geq 3$.

The $\vee$-irreducibles are easily described. In the following theorem, $A$ may be an arbitrary, not necessarily finite, set.

4.1 Theorem. The completely $\vee$-irreducibles of $\mathcal{E}$ are exactly the congruence lattices of the form

$$
E_{\varkappa}:=\operatorname{Con}(A, \text { End } \varkappa)=\{\Delta, \varkappa, \nabla\}
$$

where $\varkappa \in \operatorname{Eq}(A) \backslash\{\Delta, \nabla\}$ is an arbitrary equivalence relation. Moreover, each $\vee$-irreducible is an atom in $\mathcal{E}$, i.e. the lattice $\mathcal{E}$ is atomistic.

Proof. Completely $\vee$-irreducibles must be of the form $E_{\varkappa}$ as noted in 2.2. The characterization $(*)$ follows immediately from [PösR08, Corollary 2.5], where it is shown that $\operatorname{Quord}(A$, End $\varkappa)=\{\Delta, \varkappa, \nabla\}$ (and therefore equals $\operatorname{Con}(A$, End $\varkappa))$ for any equivalence relation $\varkappa$. Clearly, such lattices are atoms in $\mathcal{E}$ and therefore $\vee$-irreducible, since $\{\Delta, \nabla\}$ is the only proper sublattice.

4.2 Remark. For $L \in \mathcal{E}$ let $\operatorname{At}(L):=\left\{E_{\varkappa} \mid E_{\varkappa} \subseteq L, \varkappa \in \operatorname{Eq}(A)\right\}=\left\{E_{\varkappa} \mid\right.$ $\varkappa \in L \backslash\{\Delta, \nabla\}\}$ be the set of atoms contained in $L$. It is natural to ask which sets of atoms are of the form $\operatorname{At}(L)$ for some $L \in \mathcal{E}$. Equivalently, for given $E \subseteq \operatorname{Eq}(A)$, we may ask for $\operatorname{At}(\operatorname{Con}(A, \operatorname{End} E))$. Formally we put $[E]:=$ $\{\Delta, \nabla\} \cup \operatorname{At}(\operatorname{Con}(A$, End $E))$ because then $E \mapsto[E]$ is a closure operator which is well-known: $[E]$ coincides with the Galois closures of the Galois connection End - Inv as well as of Pol - Inv restricted to equivalence relations (because Con $F=\operatorname{Inv} F \cap \mathrm{Eq}(A))$, cf. [PösK79] or [Pös04], and can be explicitly described by so-called graphical compositions as shown by H. WeRner in [Wer76].

Now we describe the coatoms.

4.3 Theorem. The coatoms of $\mathcal{E}$ are exactly the congruence lattices of the form $\operatorname{Con}(A, f)$ where $f \in A^{A}$ satisfies

(I) $f$ is nontrivial and $f^{2}=f$, or 
(II) $f$ is nontrivial, $f^{2}$ is a constant, say 0 , and $\left|[0]_{\operatorname{ker} f}\right| \geq 3$, or

(III) $f^{p}=\operatorname{id}_{A}$ for some prime $p$ such that the permutation $f$ has at least two cycles of length $p$.

Remark: It can happen that different functions $f$ from the theorem give the same coatom. This explicitly will be clarified in Proposition 4.8. The theorem describes three types of functions, the graphs of which are shown in Figure 1 (all labeled elements are mandatory, all others are optional). Moreover, there are functions which define coatoms but which are of none of the types (I)-(III), e.g., on $A=\{0,1,2\}$ we get a coatom $\operatorname{Con}(A, f)=\operatorname{Con}(A, g)$ for $f: 1 \mapsto 0 \mapsto 0$, $2 \mapsto 2$ and $g: 1 \mapsto 2 \mapsto 0 \mapsto 2$ where $f$ is of type (I), but $g$ is of no type.

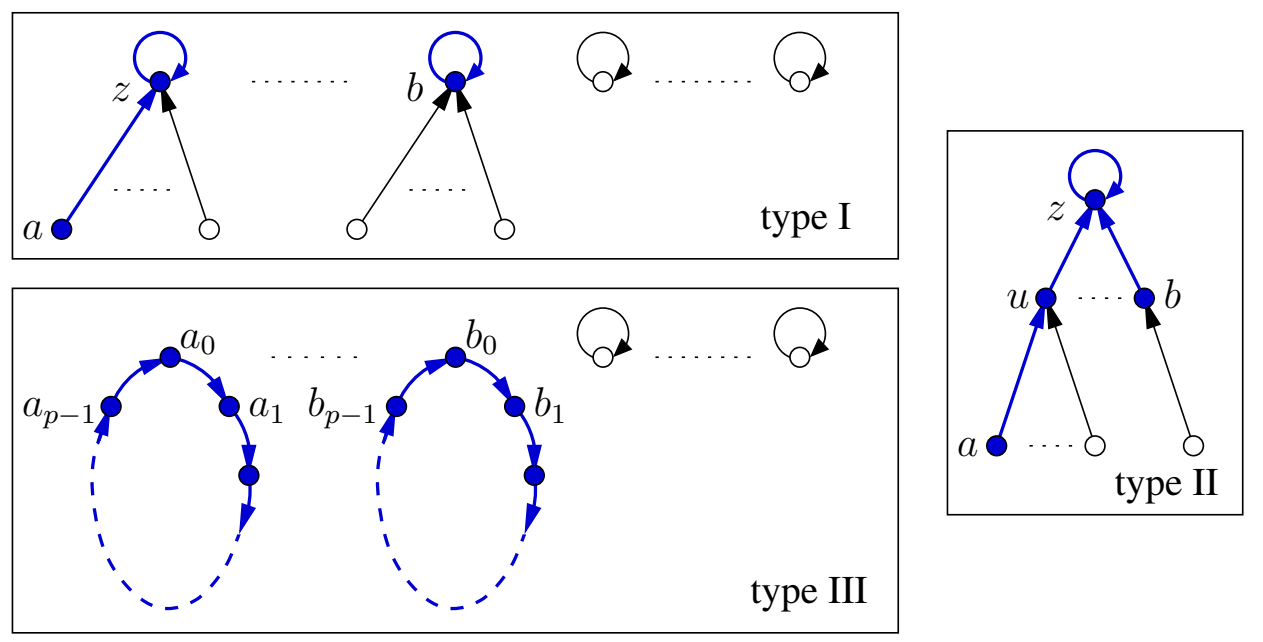

Figure 1: The graphs of functions of type I, II and III

Proof. The coatoms in the lattice $\mathcal{L}$ of quasiorder lattices are known from [JakPR16, Theorem 3.1] and can be described exactly as Quord $(A, f)$ for the nontrivial functions $f$ of the three types (I)-(III). Thus the Theorem immediately follows from Corollary 3.5.

Note that the proof is based on Corollary 3.5 which follows from 3.1(iv) and needs the condition $(\ddagger)$ in its concrete form in Lemma 3.4(ii). This will be proved with Proposition 4.8 below. Moreover, in 4.8 it will be clarified when two different functions of type (I)-(III) give the same congruence lattice $\operatorname{Con}(A, f)$ (by 3.1(iii) and 3.4(i) we already know that each coatom in $\mathcal{E}$ must be of the form $\Phi(Q)=\operatorname{Con}(A, f)$ for some function $f$ of type (I)-(III)). However, before stating 4.8 we need some more notions, notations and a lemma.

4.4. If $f \in A^{A}$ is of type (I) and has exactly one nontrivial component $K_{f}(z)$ with fixed point $z$, then let $\hat{f}$ be defined by

$$
\hat{f} x:= \begin{cases}z & \text { if } f x=x \\ x & \text { otherwise }\end{cases}
$$


see Figure 2. If $f \in A^{A}$ is of type (II) with two-element image $f[A]=\{u, z\}$, where $z$ shall denote the fixed point, then let $\hat{f}$ be defined by

$$
\hat{f} x:= \begin{cases}z & \text { if } f x=u \\ u & \text { otherwise. }\end{cases}
$$

In all other cases we put $\hat{f}:=f$. From the Figure 2 is clear that $\hat{\hat{f}}=f$, moreover $(A, f)$ and $(A, \hat{f})$ have the same principal congruences, thus $\operatorname{Con}(A, f)=$ $\operatorname{Con}(A, \hat{f})$.
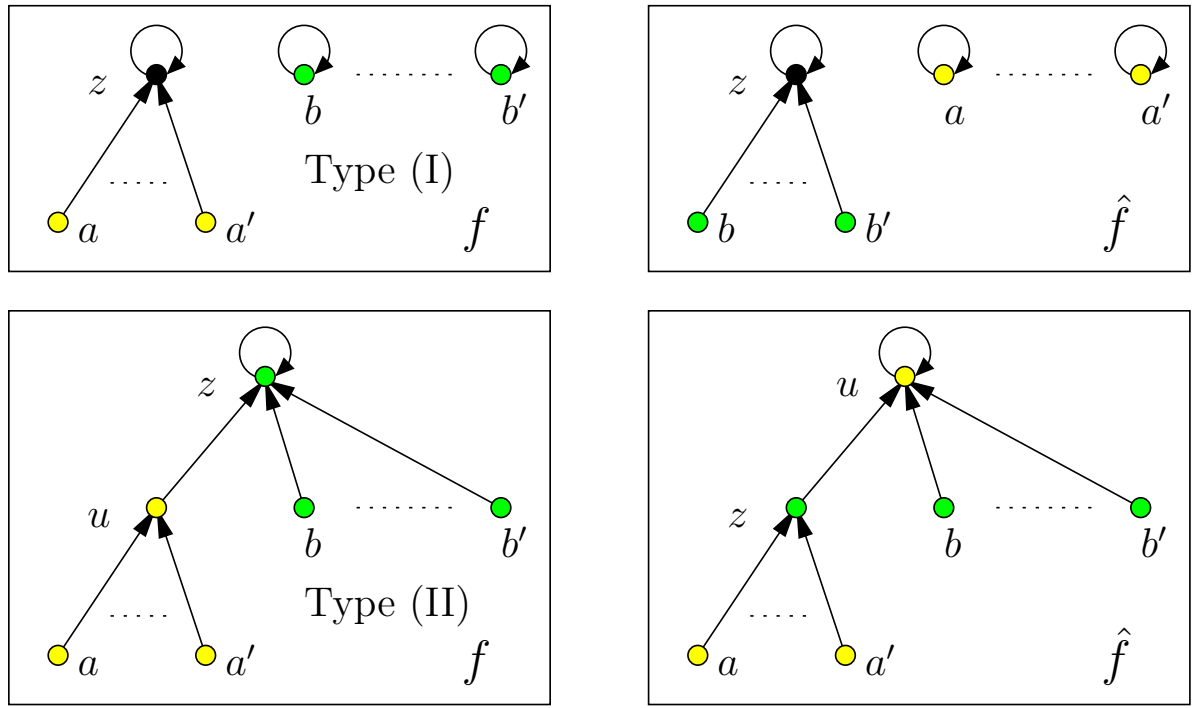

Figure 2: The functions $\hat{f}$

4.5 Definition. For a function $g \in A^{A}$ of type (I) or (II), respectively, a triple $(x, z, y)$ of three different elements is called essential for $g$ (or $g$-essential) if $g x=z=g z$ and $g y=y$, or $g x=y$ and $g y=z=g z$, respectively, see Figure 3 .

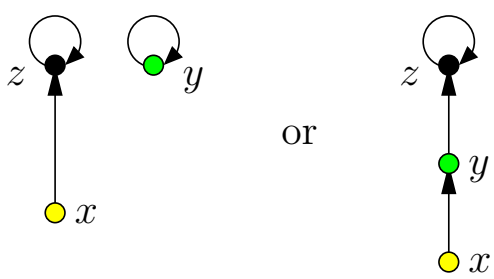

Figure 3: The graph of $g \uparrow_{\{x, y, z\}}$ for a $g$-essential triple $(x, z, y)$

4.6 Lemma. Let $\operatorname{Con}(A, f) \subseteq \operatorname{Con}(A, g)$ for functions $f, g \in A^{A}$, and let three different elements $x, y, z \in A$ satisfy $(x, z) \in \theta_{g}(x, y)$. Then we have:

(a) If $f$ is of type (I), then $(x, z, y)$ or $(y, z, x)$ is $f$-essential. 
Jakubíková-Studenovská/Pöschel/Radeleczki, Lattice of congruence lattices 11

(b) If $f$ is of type (II), then $(x, y, z)$ or $(x, z, y)$ or $(y, x, z)$ or $(y, z, x)$ is $f$ essential (note that $z$ never appears in the first component of these triples).

Proof. Because of Lemma 2.5(i) we have $(x, z) \in \theta_{g}(x, y) \subseteq \theta_{f}(x, y)$. For functions of type (I) or (II) the only possibilities that all three elements $x, y, z$ belong to the same block of $\theta_{f}(x, y)$ are those mentioned in (a) and (b), cf. Figure 3 and Figure 1.

4.7 Remark. If $g \in A^{A}$ is of type (I) or (II), then the $g$-essential triples $(x, z, y):=(a, z, b)$ or $(x, z, y):=(a, z, u)$ (notation as in Figure 1), respectively, satisfy the condition $(x, z) \in \theta_{g}(x, y)$ from Lemma 4.6.

4.8 Proposition. Let $f, g \in A^{A}$ be nontrivial operations of one of the types (I)-(III) such that $\operatorname{Con}(A, f) \subseteq \operatorname{Con}(A, g)$. Then $g \in\{f, \hat{f}\}$ if $f$ is of type (I) or (II), and $g=f^{i}$ for some $i \in\{1, \ldots, p-1\}$ if $f$ is of type (III). In particular we always have $\operatorname{Con}(A, f)=\operatorname{Con}(A, g)$.

Proof. If $f$ is of type (III), then from Proposition 2.8 (where functions $f$ of prime power order $p^{m}$ are considered, here one has to take $m=1$ ) we conclude that $g=f^{k}$ for some $k \in\{1, \ldots, p-1\}$. Corresponding to the types of $f$ and $g$, there remain altogether 6 cases to consider, denoted by $(X, Y)$ if $f$ is of type $(X)$ and $g$ of type $(Y)$, where $X \in\{\mathrm{I}, \mathrm{II}\}$ and $Y \in\{\mathrm{I}, \mathrm{II}, \mathrm{III}\}$. We start with the cases where $f$ and $g$ have different types. For these cases we shall indicate elements $x, y \in A$ with $\theta_{g}(x, y) \nsubseteq \theta_{f}(x, y)$, a contradiction to 2.5(i); e.g. by using Lemma 4.6 or by finding $(x, z) \in \theta_{g}(x, y) \backslash \theta_{f}(x, y)$.

Case (I, II): Take the $g$-essential triple $(x, z, y)=(a, z, u)$. By 4.6(a) we have that $(a, z, u)$ or $(u, z, a)$ is $f$-essential. Thus $(a, u) \notin \theta_{f}(a, z)=[a, z]$, in contradiction to $(a, u) \in \theta_{g}(a, z) \subseteq \theta_{f}(a, z)$.

Cases (I, III) and (II, III): Since $g$ is of type (III), there exist elements $a_{0}, b_{0}$ with $\theta_{g}\left(a_{0}, b_{0}\right)=\left[a_{0}, b_{0}\right]\left[a_{1}, b_{1}\right] \ldots\left[a_{p-1}, b_{p-1}\right]$ (see Figure 1). Because $\theta_{g}\left(a_{0}, b_{0}\right) \subseteq$ $\theta_{f}\left(a_{0}, b_{0}\right)$ we get that $f$ (as function of type (I) or (II)) must satisfy $\left\{f a_{0}, f b_{0}\right\}=$ $\left\{a_{1}, b_{1}\right\}$ (moreover, $p=2$ ) and $\theta_{f}\left(a_{1}, b_{1}\right)=\left[a_{1}, b_{1}\right]$ (because $f^{2}=f$ for type (I), and $f^{2} a_{0}=f^{2} b_{0}=z$ for $f$ of type (II)); in particular $\theta_{f}\left(a_{1}, b_{1}\right)$ cannot contain $\theta_{g}\left(a_{1}, b_{1}\right)=\theta_{g}\left(a_{0}, b_{0}\right)$, a contradiction.

Case (II, I): Take the $g$-essential triple $(x, z, y):=(a, z, b)$. By $4.6(\mathrm{~b})$ there exists an $f$-essential triple $\left(a^{\prime}, z^{\prime}, u^{\prime}\right)$ with $\{a, b, z\}=\left\{a^{\prime}, u^{\prime}, z^{\prime}\right\}$. Further, there must exist $b^{\prime} \notin\left\{a^{\prime}, u^{\prime}, z^{\prime}\right\}$ with $f b^{\prime}=z^{\prime}$. Note that $\theta_{f}\left(x, b^{\prime}\right)$ consists only of 2element blocks for each $x \in\left\{a^{\prime}, u^{\prime}, z^{\prime}\right\}=\{a, b, z\}$. Therefore, $\theta_{g}\left(a, b^{\prime}\right)=\left[a, b^{\prime}, z\right]$ if $g b^{\prime}=b^{\prime}$, or $\theta_{g}\left(b, b^{\prime}\right)=\left[b, b^{\prime}, g b^{\prime}\right]$ if $g b^{\prime} \neq b^{\prime}$, cannot be contained in $\theta_{f}\left(a, b^{\prime}\right)$ or $\theta_{f}\left(b, b^{\prime}\right)$, respectively, a contradiction.

Now we continue with the cases where $f$ and $g$ are of the same type.

Case (I, I): Let $(a, z, b)$ be an $g$-essential triple. According to Lemma 4.6(a), we have only the following two possibilities:

$$
\begin{gathered}
f a=z \text { and } f b=b \\
\text { or } f b=z \text { and } f a=a .
\end{gathered}
$$


Jakubíková-Studenovská/Pöschel/Radeleczki, Lattice of congruence lattices 12

Clearly this must hold for each essential triple of $g$. We show that $\left(^{*}\right)$ implies $g=f$ and $\left({ }^{*}\right)$ implies $f=\hat{g}$. Let $c \in A \backslash\{a, b, z\}$ arbitrary.

Consider case $(*)$ : If $g c=c$, take the $g$-essential triple $(a, z, c)$ for which only case $\left({ }^{*}\right)$ is possible (since $f a=z$ ), i.e. $f c=c=g c$. If $g c \neq c$, take the $g$-essential triple $(c, g c, b)$ (provided that $g c \neq b)$ or $(c, g c, z)$ (if $g c=b$ ) for which again case $\left(^{*}\right)$ must hold (since $f b=b$ and $f z=z$ ), i.e. $f c=g c$, too. Altogether $f=g$.

Now consider case $(* *)$ : If $g c=c$, take the $g$-essential triple $(a, z, c)$ for which only case $\left({ }^{*}\right)$ is possible ( since $f a=a$ ), i.e. $f c=z$. If $g c=z \neq c$, take the essential triple $(c, z, b)$ for which again case $(* *)$ must hold (since $f b=z$ ), i.e. $f c=c$. The case $g c \notin\{c, z\}$ cannot appear because then we would have $[a, c][z, g c]=\theta_{g}(a, c) \subseteq \theta_{f}(a, c)=[a, c, f c]$, a contradiction. Thus altogether we have $f=\hat{g}$.

Case (II, II): Let $(a, z, u)$ be a $g$-essential triple. Then the triples $\left(x, z^{\prime}, y\right)=$ $\overline{(a, z, u) \text { and }}\left(x, z^{\prime}, y\right)=(a, u, z)$ satisfy the assumptions of Lemma 4.6(b), consequently neither $z$ nor $u$ can be in the first component of the corresponding $f$-essential triples. Thus there remain only the following two possibilities:

$$
\begin{aligned}
f a & =u \text { and } f u=z=f z \\
\text { or } f a & =z \text { and } f z=u=f u .
\end{aligned}
$$

Clearly this must hold for each essential triple of $g$. We show that $\left(^{*}\right)$ implies $g=f$ and $(* *)$ implies $f=\hat{g}$ (equivalentyl, $g=\hat{f}$ ).

Consider case $\left(^{*}\right)$ : Since $f z=z$ is the unique fixed point of $f$, for each $g$ essential triple $\left(a^{\prime}, z, u^{\prime}\right)$ we get case $(*)$,i.e. $\left(a^{\prime}, z, u^{\prime}\right)$ is $f$-essential and thus $f$ and $g$ agree on all essential triples. If $b$ does not belong to an $g$-essential triple, then $g b=z$ and $\theta_{g}(a, b)=[a, b][u, z]$. From $\theta_{g}(a, b) \subseteq \theta_{f}(a, b)=[a, b][u, f b]$ we conclude $f b=z$; altogether $f=g$.

Consider now case $(* *)$ : Since $f u=u$ is the unique fixed point of $f$ but $f z=u$ is not a fixed point, for each $g$-essential triple $\left(a^{\prime}, z, u^{\prime}\right)$ we must have case $\left.{ }^{* *}\right)$, i.e. $\left(a^{\prime}, u^{\prime}, z\right)$ is $f$-essential, in particular $u^{\prime}=u$ and $f a^{\prime}=z$. If $b$ does not belong to an $g$-essential triple, then $g b=z$ and $\theta_{g}(a, b)=[a, b][u, z]$. From $\theta_{g}(a, b) \subseteq \theta_{f}(a, b)=[a, b][z, f b]$ we conclude $f b=u$; altogether $f=\hat{g}$.

\section{$5 \wedge$-irreducible $\operatorname{Con}(A, f)$ in $\mathcal{E}$ with permutation $f$}

The coatoms are $\wedge$-irreducible in $\mathcal{E}$. Now we want to deal with $\wedge$-irreducible congruence lattices in general. They all must be of the form $\operatorname{Con}(A, f)$ for a single $f$ and we have:

5.1 Proposition. Let $\operatorname{Con}(A, f)$ be a $\wedge$-irreducible element in $\mathcal{E}$. Then there exists $g \in A^{A}$ such that $\operatorname{Quord}(A, g)$ is $\wedge$-irreducible in $\mathcal{L}$ and $\operatorname{Con}(A, f)=$ $\operatorname{Con}(A, g), \operatorname{Quord}(A, f) \subseteq \operatorname{Quord}(A, g)$.

Proof. The proof directly follows from Proposition 3.1(i) and Remark 3.2 applied to the residual mapping $\Phi$, cf. 3.3 (the role of $m, q, r$ in 3.1(i) and 3.2 here 
is played by $\operatorname{Con}(A, f), \operatorname{Quord}(A, g)$, Quord $(A, f))$.

We shall describe first the $\wedge$-irreducibles for permutations $f$ and - in the next section - for acyclic $f$.

For permutations $f$ the $\wedge$-irreducible quasiorder lattices Quord $(A, f)$ are known. They are described in [JakPR16, Theorem 3.2]: $f$ is either a transposition or of the form as given in Theorem 5.4 below. We first exclude the transpositions:

5.2 Lemma. Let $f \in A^{A}$ be a transposition $(|A| \geq 3)$. Then $\operatorname{Con}(A, f)$ is not $\wedge$-irreducible.

Proof. If $f \in A^{A}$ is a transposition, then there are elements $0,1 \in A$ such that $f 0=1, f 1=0$ and $f x=x$ for $x \in A \backslash\{0,1\}$. Let $g_{0}, g_{1} \in A^{A}$ be the nontrivial functions defined by $g_{0} 0=g_{0} 1=0, g_{1} 0=g_{1} 1=1$ and all $x \in A \backslash\{0,1\}$ are fixed point for $g_{0}$ and $g_{1}$. Then, for the principal congruences, we have $\theta_{f}(x, y)=\theta_{g_{0}}(x, y)=\theta_{g_{1}}(x, y)=[x, y]$ for all $x, y \in A$ with the only exceptions $\theta_{f}(0, x)=\theta_{f}(1, x)=\theta_{g_{0}}(1, x)=\theta_{g_{1}}(0, x)=[0,1, x]$ for all $x \in A \backslash\{0,1\}$. Note $\theta_{f}(0, x) \neq \theta_{g_{0}}(0, x)$ and $\theta_{f}(1, x) \neq \theta_{g_{1}}(1, x)$. Therefore $\operatorname{Con}(A, f) \varsubsetneqq \operatorname{Con}\left(A, g_{i}\right)$, $i \in\{0,1\}$ and $\operatorname{Con}(A, f)=\operatorname{Con}\left(A, g_{0}\right) \cap \operatorname{Con}\left(A, g_{1}\right)$.

The following proposition deals with those functions which will play the crucial role in the next theorem.

5.3 Proposition. Let $|A| \geq 3$ and let $f \in \operatorname{Sym}(A)$ be a permutation of prime power order $p^{m}$ with at least two cycles of length $p^{m}$. Then the principal filter

$$
[\operatorname{Con}(A, f)\rangle_{\mathcal{E}}:=\{E \in \mathcal{E} \mid \operatorname{Con}(A, f) \subseteq E\}
$$

is a chain. Moreover, each element of this chain is $\wedge$-irreducible (except the top element $\operatorname{Eq}(A))$ and is of the form $\operatorname{Con}(A, g)$, where $g=f^{k}$ for some $k \in \mathbb{N}_{+}$.

Proof. Given $f$ as indicated we know from [JakPR16, Theorem 4.2 and 4.3] that the principal filter $[\mathrm{Quord}(A, f)\rangle_{\mathcal{L}}$ is a chain. From Lemma 2.7 and Corollary 3.7 we conclude $\Phi\left([\operatorname{Quord}(A, f)\rangle_{\mathcal{L}}\right)=[\operatorname{Con}(A, f)\rangle_{\mathcal{E}}$, consequently $[\operatorname{Con}(A, f)\rangle_{\mathcal{E}}$ is also a chain, therefore each element (except $\operatorname{Eq}(A))$ of this chain is $\wedge$-irreducible and thus of the form $\operatorname{Con}(A, g)$. By 2.8 each nontrivial $g$ with $\operatorname{Con}(A, f) \subseteq$ $\operatorname{Con}(A, g)$ is of the form $g=f^{k}$.

5.4 Theorem. A congruence lattice $\operatorname{Con}(A, f)$ with a nontrivial permutation $f$ is $\wedge$-irreducible in $\mathcal{E}$ if and only if $f$ is of prime power order $p^{m}$ with at least two cycles of length $p^{m}$.

Proof. " $\Leftarrow$ " was proved in 5.3 .

" $\Rightarrow$ ": Let $f$ be a permutation such that $\operatorname{Con}(A, f)$ is $\wedge$-irreducible. By 5.1 there exists $g \in A^{A}$ such that $\operatorname{Quord}(A, g)$ is $\wedge$-irreducible in $\mathcal{L}$ and

$$
(*) \operatorname{Quord}(A, f) \subseteq \operatorname{Quord}(A, g), \quad(* *) \operatorname{Con}(A, f)=\operatorname{Con}(A, g) \text {. }
$$


Jakubíková-Studenovská/Pöschel/Radeleczki, Lattice of congruence lattices 14

As shown in [JakPR16, Lemma 2.4(iv)]), from $(*)$ follows that each subalgebra of $(A, f)$ is also a subalgebra of $(A, g)$, while from (**) and Lemma 2.5(ii) (interchange the roles of $f$ and $g$ ) follows that each subalgebra of $(A, g)$ with at least two elements is also a subalgebra of $(A, f)$ (since $f$ is a permutation, it cannot be constant on two elements).

At first we show that $g$ is also a permutation. Let $x, y \in A$ such that $x \neq y$. Since cycles (what coincides with components) of $f$ are subalgebras of $(A, f)$ and thus also of $(A, g), K_{f}(x) \neq K_{f}(y)$ implies $g x \in K_{f}(x), g y \in K_{f}(y)$, hence $g x \neq g y$. Thus let $x, y$ belong to the same cycle of $f$ and assume $g x=g y$. Then from (**) and Lemma 2.5(i) (here the roles of $f$ and $g$ are interchanged) we conclude $(f x, f y) \in \theta_{g}(x, y)=[x, y]$, thus $\{f x, f y\}=\{x, y\}$ is a subalgebra of $(A, f)$ and therefore $\{x, y\} \leq(A, g)$ (as mentioned above). Thus w.l.o.g. we can assume $g x=g y=x$. Let $z \in A \backslash\{x, y\}$ and let $C$ be the cycle of $f$ which contains $z$. Then $\{x\} \cup C$ is a subalgebra(with at least 2-elements) of $(A, g)$ but not of $(A, f)$ (since $f x=y \notin\{x\} \cup C)$, a contradiction.

Thus $g$ is a permutation. Therefore, from [JakPR16, Proposition 2.5(b)] and $\wedge$-irreducibility of $\operatorname{Quord}(A, g)$ we get that $g$ is a permutation of prime power order $p^{m}$ with at least two cycles of length $p^{m}$ or that $g$ is a transposition. Since $\operatorname{Con}(A, g)=\operatorname{Con}(A, f)$ is $\wedge$-irreducible, $g$ cannot be a transposition by Lemma 5.2. From $(* *)$ and Proposition 2.8 (interchange the role of $f$ and $g$ ) we get $f=g^{k}$ for some $k \in \mathbb{N}_{+}$. From $(* *)$ we further conclude that $p$ cannot divide $k$ (since $\operatorname{Con}\left(A, g^{p}\right) \supsetneqq \operatorname{Con}(A, g)$ ). Therefore $f$ and $g$ generate the same cyclic subgroup, in particular $f$ also has order $p^{m}$ and at least two cycles of length $p^{m}$, and we are done.

\section{$6 \wedge$-irreducible $\operatorname{Con}(A, f)$ in $\mathcal{E}$ with acyclic $f$}

In this section we deal with acyclic algebras $(A, f)$. For an acyclic $f \in A^{A}$ and $x \in A$ let $t_{f}(x):=\min \left\{n \in \mathbb{N} \mid f^{n} x=f^{n+1} x\right\}$ denote the so-called depth of $x$ (after $n$ times applying $f$ to $x$ one gets a fixed point) and let

$$
\bar{t}(F):=\max \left\{t_{f}(x) \mid x \in A\right\}
$$

(this is the length of a longest "tail" in the graph of $f$ ).

6.1. For a nontrivial, acyclic function $f \in A^{A}$ we consider the following conditions (cf. Figure 4):

(a) There exist distinct elements $0,1,2,0^{\prime}, 1^{\prime}, 2^{\prime} \in A$ such that $f 2=1, f 1=$ $f 0=0$ and $f 2^{\prime}=1^{\prime}, f 1^{\prime}=f 0^{\prime}=0^{\prime}$,

(b) $f$ is connected (i.e., only one component) and there exist distinct elements $0,1,2,1^{\prime}, 2^{\prime} \in A$ such that $f 2=1, f 2^{\prime}=1^{\prime}, f 1^{\prime}=f 1=f 0=0$.

6.2 Proposition. Let $f \in A^{A}$ be nontrivial and acyclic such that $f$ is not of type (I), not of type (II) and satisfies neither condition 6.1(a) nor (b). Then $\operatorname{Con}(A, f)$ is $\wedge$-reducible. 


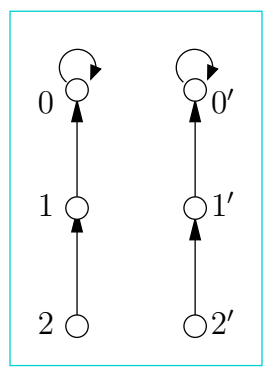

(a)

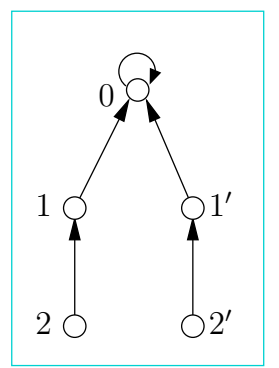

(b)

Figure 4: The action of $f$ on the elements in conditions $6.1(\mathrm{a}),(\mathrm{b})$

Proof. If $\bar{t}(f)=1$ then $f$ is of type (I). Thus we can assume $\bar{t}(f) \geq 2$. We distinguish the following cases:

Case 1: $f$ has at least two components.

Then (b) trivially does not hold. If (a) fails to hold, then $f$ has exactly one component, say $K$, with elements of depth 2 while all other components have elements of depth at most 1 . In particular there are at least two fixed points, say $0 \in K$ and $0^{\prime}$. Therefore $f$ is of the form as given in Figure 5 (the shadowed part is $K)$.
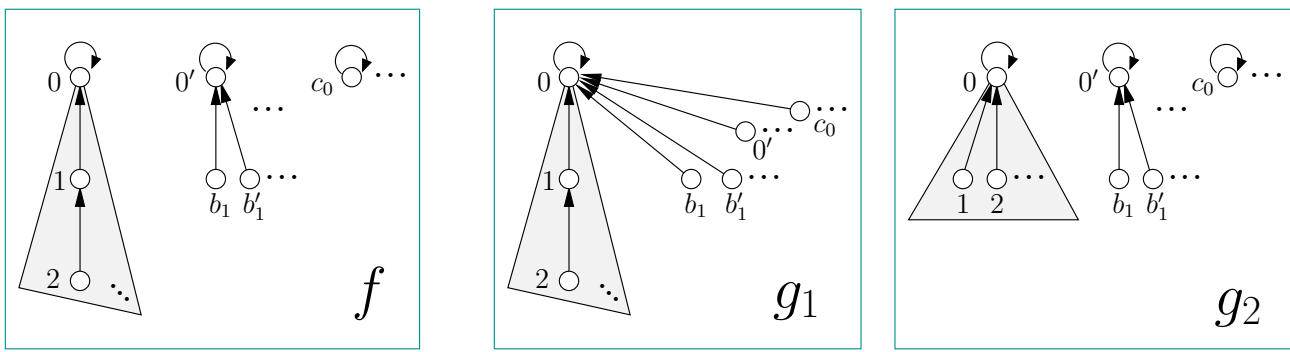

Figure 5: Functions $f, g_{1}, g_{2}$ with $\operatorname{Con}(A, f)=\operatorname{Con}\left(A, g_{1}\right) \cap \operatorname{Con}\left(A, g_{2}\right)$

We define the functions $g_{1}$ and $g_{2}$ as follows (see Figure 5):

$$
g_{1} x:=\left\{\begin{array}{ll}
f x & \text { if } x \in K, \\
0 & \text { otherwise }
\end{array} \quad g_{2} x:= \begin{cases}0 & \text { if } x \in K \\
f x & \text { otherwise }\end{cases}\right.
$$

Case 2: $f$ has only one component with fixed point, say 0.

Then (a) trivially does not hold. If (b) fails to hold then all elements $x$ with $t_{f}(x)=2$ map to the same element, say 1 , of depth 1 .

Case 2a: If $\bar{t}(f)=2$, then there is only one element of depth 1 , because otherwise $f$ would be of type (II) what is excluded by assumption. Therefore $f$ is of the form as given in Figure 6.

We define the functions $g_{1}$ and $g_{2}$ as follows (see Figure 6):

$$
g_{1} x:=\left\{\begin{array}{ll}
0 & \text { if } x=0, \\
1 & \text { otherwise. }
\end{array} \quad g_{2} x:= \begin{cases}1 & \text { if } x=1, \\
0 & \text { otherwise }\end{cases}\right.
$$


Jakubíková-Studenovská/Pöschel/Radeleczki, Lattice of congruence lattices 16
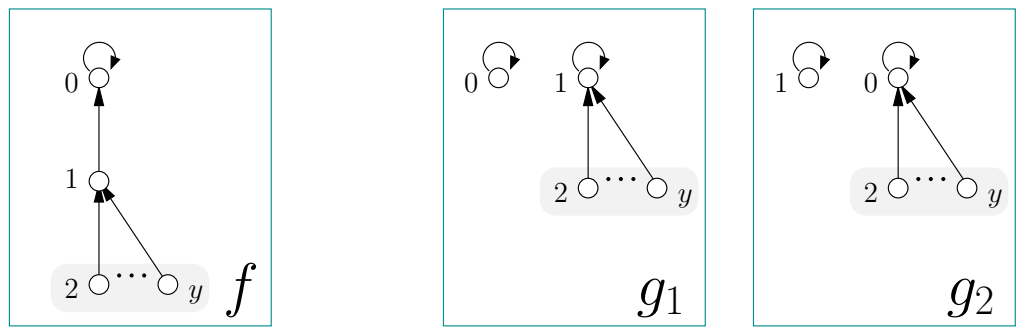

Figure 6: Functions $f, g_{1}, g_{2}$ with $\operatorname{Con}(A, f)=\operatorname{Con}\left(A, g_{1}\right) \cap \operatorname{Con}\left(A, g_{2}\right)$

Case 2b: If $\bar{t}(f) \geq 3$ then $f$ must be of the form as given in Figure 7 .
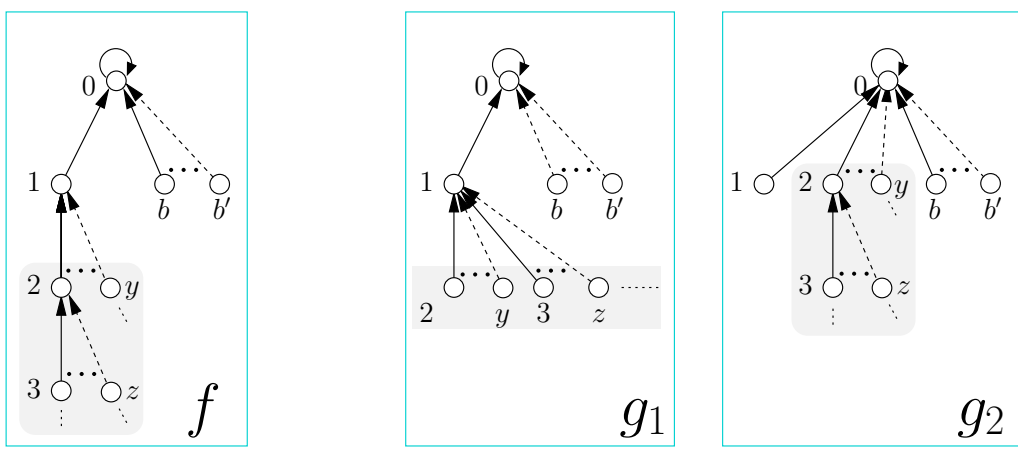

Figure 7: Functions $f, g_{1}, g_{2}$ with $\operatorname{Con}(A, f)=\operatorname{Con}\left(A, g_{1}\right) \cap \operatorname{Con}\left(A, g_{2}\right)$

We define the functions $g_{1}$ and $g_{2}$ as follows (see Figure 7):

$$
g_{1} x:=\left\{\begin{array}{ll}
1 & \text { if } t_{f}(x) \geq 2, \\
0 & \text { otherwise }
\end{array} \quad g_{2} x:= \begin{cases}0 & \text { if } t_{f}(x)=2 \\
f x & \text { otherwise }\end{cases}\right.
$$

In all cases the functions $g_{1}, g_{2}$ are nontrivial and it is easy to check that these functions satisfy $\operatorname{Con}(A, f) \varsubsetneqq \operatorname{Con}\left(A, g_{i}\right)(i \in\{1,2\})$ and $\operatorname{Con}(A, f)=$ $\operatorname{Con}\left(A, g_{1}\right) \cap \operatorname{Con}\left(A, g_{2}\right)$. In fact, using the Figures 5, 6 and 7 , we can check $\left(g_{i} x, g_{i} y\right) \in \theta_{f}(x, y)$, therefore $\operatorname{Con}(A, f) \subseteq \operatorname{Con}\left(A, g_{i}\right)$ by Remark 2.6; moreover the inclusions are strict (e.g. for Case 1 we have $\left[1, b_{1}\right]=\theta_{g_{1}}\left(1, b_{1}\right) \neq \theta_{f}\left(1, b_{1}\right)=$ $\left.\left[1, b_{1}\right]\left[0,0^{\prime}\right]\right)$. Further, $\theta_{f}(x, y)=\theta_{g_{1}}(x, y) \vee \theta_{g_{2}}(x, y)$ for all $x, y \in A$ (e.g. for Case 1 we have $\theta_{f}\left(2, b_{1}\right)=\left[2, b_{1}\right]\left[1,0,0^{\prime}\right]=\left[2, b_{1}\right][1,0] \vee\left[2, b_{1}\right]\left[0,0^{\prime}\right]=\theta_{g_{1}}\left(2, b_{1}\right) \vee$ $\theta_{g_{2}}\left(2, b_{1}\right)$, cf. Figure 5). Consequently, $\operatorname{Con}(A, f)=\operatorname{Con}\left(A, g_{1}\right) \cap \operatorname{Con}\left(A, g_{2}\right)$. Thus $(A, f)$ is $\wedge$-reducible.

6.3 Proposition. Let $f \in A^{A}$ be acyclic such that $f$ is not of type (I), not of type (II) and does satisfy either condition $6.1(\mathrm{a})$ or (b). Let $\operatorname{Con}(A, f) \varsubsetneqq$ $\operatorname{Con}(A, g)$ for $g \in A^{A}$. Then we have $\varrho_{1} \in \operatorname{Con}(A, g)$ for the equivalence relation $\varrho_{1}:=[0,2]$.

Proof. Assume $\varrho_{1} \notin \operatorname{Con}(A, g)$ and we shall show that this leads to a contradiction. If $f$ satisfies (b), it is convenient to put formally $0^{\prime}:=0$.

We have $\bar{t}(f) \geq 2$, because $\bar{t}(f)=1$ means that $f$ is of type (I). 
Claim 1: $\{0,1,2\} \leq(A, g)$.

In fact, if $\{0,1,2\}$ were not a subalgebra, then (by 2.5 (ii) $) g$ would be constant on $\{0,1,2\}$. Thus $\varrho_{1}=[0,2]=\theta_{g}(0,2) \in \operatorname{Con}(A, g)$, a contradiction.

Claim 2: $\{0,1\} \leq(A, g)$.

Assume that $\{0,1\}$ is not a subalgebra. Then $g$ must be constant on $\{0,1\}$ (by $2.5(\mathrm{ii})$ ) where the constant is outside $\{0,1\}$. Because of Claim 1 we get $g 0=g 1=2$ and $g 2 \in\{0,1,2\}$. The values $g 2 \in\{0,2\}$ cannot appear (otherwise $\left.\varrho_{1}=[0,2] \in \operatorname{Con}(A, g)\right)$, thus it remains $g 2=1$. Since $\left\{0,0^{\prime}, 1,1^{\prime}\right\} \leq(A, f)$, again by 2.5 (ii) $g$ must be constant 2 on these elements, in particular $g 1^{\prime}=2$. Consequently $(1,2)=\left(g 2, g 1^{\prime}\right) \in \theta_{f}\left(2,1^{\prime}\right)=\left[2,1^{\prime}\right]\left[1,0^{\prime}, 0\right]$, a contradiction (see Figure 4).

Claim 3: We have $\{0\} \leq(A, g)$, i.e. $g 0=0$.

If $g 0 \neq 0$, then $g 0=1$ (by Claim 2). Because $1 \notin\left\{0,0^{\prime}, 1^{\prime}, 2^{\prime}\right\} \leq(A, f)$ (recall $0^{\prime}=0$ in case (b)) and by 2.5(ii) $g$ must be constant 1 on these elements, in particular $g 2^{\prime}=1$. Thus $(g 2,1)=\left(g 2, g 2^{\prime}\right) \in \theta_{f}\left(2,2^{\prime}\right)=\left[2,2^{\prime}\right]\left[1,1^{\prime}\right]\left[0,0^{\prime}\right]$ implies $g 2=1$ according to Claim 1 . Consequently $\theta_{g}(0,2)=[0,2]=\varrho_{1}$, a contradiction. Thus $g 0=0$.

Claim 4: $g$ and $f$ agree on $\{0,1,2\}$.

Because $g 0=0$ (by claim 3), the values $g 2 \in\{0,2\}$ cannot appear (otherwise $\left.\varrho_{1}=[0,2] \in \operatorname{Con}(A, g)\right)$. Thus $g 2=1$ (by Claim 1 ). It remains to prove $g 1=0$. If $g 1 \neq 0$ then $g 1=1$ (by Claim 2). Thus $\left(1, g 2^{\prime}\right)=\left(g 1, g 2^{\prime}\right) \in$ $\theta_{f}\left(1,2^{\prime}\right)=\left[1,2^{\prime}\right]\left[0,1^{\prime}, 0^{\prime}\right]$ what implies either $g 2^{\prime}=2^{\prime}$ or $g 2^{\prime}=1$, the former gives the contradiction $\left(1,2^{\prime}\right)=\left(g 2, g 2^{\prime}\right) \in \theta_{f}\left(2,2^{\prime}\right)=\left[2,2^{\prime}\right]\left[1,1^{\prime}\right]\left[0,0^{\prime}\right]$ and the latter gives the contradiction $(0,1)=\left(g 0, g 2^{\prime}\right) \in \theta_{f}\left(0,2^{\prime}\right)=\left[0,0^{\prime}, 1^{\prime}, 2^{\prime}\right]$. Thus $g 1=0$

Claim 5: $g$ and $f$ agree on $\left\{0^{\prime}, 1^{\prime}, 2^{\prime}\right\}$.

From $\left(1, g 2^{\prime}\right)=\left(g 2, g 2^{\prime}\right) \in \theta_{f}\left(2,2^{\prime}\right)=\left[2,2^{\prime}\right]\left[1,1^{\prime}\right]\left[0,0^{\prime}\right]$ we conclude $g 2^{\prime} \in\left\{1,1^{\prime}\right\}$. However $g 2^{\prime}=1$ gives the contradiction as seen in Claim 4. Consequently $g 2^{\prime}=$ $1^{\prime}$. Thus $\left\{0^{\prime}, 1^{\prime}, 2^{\prime}\right\}$ is a subalgebra of $(A, g)$ (since $g 2^{\prime} \in\left\{0^{\prime}, 1^{\prime}, 2^{\prime}\right\} \leq(A, f)$ ). Further, $\left(0, g 1^{\prime}\right)=\left(g 1, g 1^{\prime}\right) \in \theta_{f}\left(1,1^{\prime}\right)=\left[1,1^{\prime}\right]\left[0,0^{\prime}\right]$ gives $g 1^{\prime} \in\left\{0^{\prime}, 0\right\}$, thus $g 1^{\prime}=0^{\prime}=f 1^{\prime}$ (note $g 1^{\prime}$ must belong to the subalgebra $\left\{0^{\prime}, 1^{\prime}, 2^{\prime}\right\}$ ). Finally, $\left(0, g 0^{\prime}\right)=\left(g 0, g 0^{\prime}\right) \in \theta_{f}\left(0,0^{\prime}\right)=\left[0,0^{\prime}\right]$ implies $g 0^{\prime}=0^{\prime}=f 0^{\prime}$ (note $g 0^{\prime}$ must belong to the subalgebra $\left.\left\{0^{\prime}, 1^{\prime}\right\}\right)$.

Claim 6: We have $Z_{f}(x) \leq(A, g)$, i.e. $Z_{g}(x) \subseteq Z_{f}(x)$, for each $x \in A$.

If $f$ satisfies $6.1(\mathrm{~b})$, then $0 \in Z_{f}(x) \leq(A, f)$, and with 2.5 (ii) and $g 0=0$ we get $Z_{f}(x) \leq(A, g)$.

If $f$ satisfies $6.1(\mathrm{a})$, then $B:=\{0\} \cup Z_{f}(x)$ and $B^{\prime}:=\left\{0^{\prime}\right\} \cup Z_{f}(x)$ are subalgebras of $(A, f)$ and $g 0=0, g 0^{\prime}=0^{\prime}$. Thus they are also subalgebras of $(A, g)$ (due to $2.5($ ii $)$ ), consequently the intersection $B \cap B^{\prime}=Z_{f}(x)$ is also a subalgebra of $(A, g)$.

Claim 7: $g=f$.

Because of Claim 4 and 5 we have to show $g x=f x$ for each $x \in A \backslash\left\{0,1,2,0^{\prime}, 1^{\prime}, 2^{\prime}\right\}$. If $x$ is a fixed point of $f$, then $Z_{f}(x)=\{x\}$ and from Claim 6 we get $g x=x=$ $f x$. Thus let $t_{f}(x) \geq 1$. W.l.o.g. we can assume $1 \notin Z_{f}(x)$ (otherwise inter- 
change the role of 1 and $1^{\prime}$ ). We have

$$
(1, g x)=(g 2, g x) \in \theta_{f}(2, x)= \begin{cases}{[2, x][1, f x]\left[0, f^{2} x, \ldots, f^{k} x\right]} & \text { if } t_{f}(x) \geq 2 \\ {[2, x][0,1, f x]} & \text { if } t_{f}(x)=1\end{cases}
$$

If $t_{f}(x) \geq 2$ we conclude $g x \in\{1, f x\}$ and get $g x=f x$ (since $1 \notin Z_{g}(x)$ ). If $t_{f}(x)=1$, then we conclude $g x \in\{0,1, f x\}$. Moreover, by assumption we have $x \notin\{0,1, f x\}$ and, by Claim 6 we get $g x \in Z_{f}(x)=\{x, f x\}$. Thus $g x \in\{x, f x\} \cap\{0,1, f x\}=\{f x\}$.

From Claim 7 we get $\operatorname{Con}(A, f)=\operatorname{Con}(A, g)$, a contradiction.

6.4 Theorem. A congruence lattice $L=\operatorname{Con}(A, f)$ with an acyclic $f \in A^{A}$ is $\wedge$-irreducible in $\mathcal{E}$ if and only if $f$ is of type (I) or (II) or satisfies the condition $6.1(\mathrm{a})$ or (b).

Proof. Note that $\operatorname{Con}(A, f)$ is a coatom for functions $f$ of type (I) or (II), and therefore $\wedge$-irreducible. So we need not consider these cases in the following.

" $\Rightarrow$ ": follows from Proposition 6.2

" $\Leftarrow$ ": From Proposition 6.3 we conclude that

$$
\varrho_{1} \in \bigcap\{\operatorname{Con}(A, g) \mid \operatorname{Con}(A, f) \varsubsetneqq \operatorname{Con}(A, g)\} .
$$

Since $\theta_{f}(0,2)=[0,1,2]$ we have $\varrho_{1} \notin \operatorname{Con}(A, f)$ and the above intersection cannot be equal to $\operatorname{Con}(A, f)$. Therefore $\operatorname{Con}(A, f)$ is $\wedge$-irreducible.

\section{$7 \quad$ Some lattice theoretical properties of $\mathcal{E}$}

At first we consider the problem how many coatoms (atoms, resp.) do we need such that their meet (join, resp.) in $\mathcal{E}$ gives the least (greatest, resp.) element of $\mathcal{E}$. We assume throughout that $|A| \geq 3$.

7.1 Proposition. There are two or three coatoms in the lattice $\mathcal{E}$ whose meet is $\mathbf{0}_{\mathcal{E}}$. More precisely, for $|A|>4$, there are two coatoms $\operatorname{Con}(A, f)$ and $\operatorname{Con}(A, g)$ such that $\operatorname{Con}(A, f) \cap \operatorname{Con}(A, g)=\{\Delta, \nabla\}$. For $|A| \leq 4$, three coatoms are necessary (and sufficient) for this property.

Proof. Since $\operatorname{Con}(A, f)=\operatorname{Eq}(A) \cap \operatorname{Quord}(A, f)$ is a coatom in $\mathcal{E}$ iff $\operatorname{Quord}(A, f)$ is a coatom in $\mathcal{L}$ (cf. 3.5, 4.8), the result mainly follows from the corresponding result in [JakPR16, Proposition 6.2]. In that paper for $|A|>5$ there are indicated two permutations $f$ and $g$ of type (III), for $|A|=5$ three permutations of type (III), for $|A|=4$ one permutation of type (III) and two functions of type (I) and for $|A|=3$ three functions of type (I). Now, with $\mathcal{E}$ instead of $\mathcal{L}$, in case $A=\{1, \ldots, n\}$ for $n=5$ also two functions (e.g., of type (II)) suffice, e.g., $f 4=2, f 5=3, f 2=f 3=f 1=1$ and $g 1=4, g 2=5, g 4=g 5=g 3=3$. It can be checked easily that two coatoms are not sufficient for $n \in\{3,4\}$. 
Jakubíková-Studenovská/Pöschel/Radeleczki, Lattice of congruence lattices 19

7.2 Proposition. There are three atoms in $\mathcal{E}$ whose join is $\mathbf{1}_{\mathcal{E}}$. More precisely, there are three equivalence relations $\varkappa_{1}, \varkappa_{2}, \varkappa_{3}$ such that $E_{\varkappa_{1}} \vee E_{\varkappa_{2}} \vee E_{\varkappa_{3}}=$ $\operatorname{Eq}(A)$.

Proof. By a result of L. Zádori [Zád86] there exist equivalence relations $\varkappa_{1}, \varkappa_{2}, \varkappa_{3}$ such that End $\left\{\varkappa_{1}, \varkappa_{2}, \varkappa_{3}\right\}=\left\{\operatorname{id}_{A}\right\}$. This implies $E_{\varkappa_{1}} \vee E_{\varkappa_{2}} \vee E_{\varkappa_{3}}=\operatorname{Con} \operatorname{End}\left(E_{\varkappa_{1}} \cup\right.$ $\left.E_{\varkappa_{2}} \cup E_{\varkappa_{3}}\right)=\operatorname{Con}\left\{\operatorname{id}_{A}\right\}=\operatorname{Eq}(A)$. (For notation $E_{\varkappa}$ see 4.1.)

Now we look for tolerances of the lattice $\mathcal{E}$. Because tolerance simplicity implies interesting properties of a lattice (see, e.g., [Kin79]), we looked for this property for the lattice $\mathcal{E}$ and got an affirmative result in Theorem 7.6 below.

At first we collect some notions, notations and facts which for clearer understanding we shall present on abstract level (for an arbitrary lattice $V$ instead of our lattice $\mathcal{E}$ ).

7.3. Let $V$ be a lattice with the order and covering relation denoted by $\leq$ and $\prec$, respectively. If $V$ is a bounded lattice (in particular, if it is finite), its least and greatest elements are denoted by $\mathbf{0}_{V}$ and $\mathbf{1}_{V}$.

A tolerance of $V$ is a reflexive and symmetric binary relation $T \subseteq V \times V$ compatible with the lattice operations $\wedge$ and $\vee$. Let $\operatorname{Tol}(V)$ denote all tolerances of $V$. With respect to set-theoretic inclusion the tolerances form an algebraic lattice $(\operatorname{Tol}(V), \cap, \sqcup)$ with least element $\Delta_{V}:=\{(x, x) \mid x \in V\}$ and greatest element $\nabla_{V}:=V \times V$ (called trivial tolerances). A lattice $V$ is called tolerance simple if it has no nontrivial tolerances, i.e., $\operatorname{Tol}(V)=\left\{\Delta_{V}, \nabla_{V}\right\}$.

For $x, y \in V$, let $T(x, y)$ denote the least tolerance in $\operatorname{Tol}(V)$ containing the pair $(x, y)$. Clearly, for each $T \in \operatorname{Tol}(V)$, we have $T=\bigsqcup\{T(x, y) \mid(x, y) \in T\}$. The following properties are known (see, e.g., [RadS05]) for $x, y \in V$ :

$$
\begin{array}{r}
T(x \wedge y, y)=T(x, x \vee y), \\
\left(\mathbf{0}_{V}, \mathbf{1}_{V}\right) \in T \in \operatorname{Tol}(V) \Longrightarrow T=\nabla_{V} .
\end{array}
$$

A lattice $V$ is called atomistic if every element $v \in V \backslash\left\{\mathbf{0}_{V}\right\}$ is the join of some atoms of $V$. The atoms of $V$, denoted by $\operatorname{At}(V)$ in the following, play an important role also in connection with tolerance simplicity. From [JanR15] we deduce (see also [JakPR16, 6.4]) the following: A finite atomistic lattice $V$ satisfying $T\left(\mathbf{0}_{V}, a\right)=\nabla_{V}$ for every atom $a \in V$, is tolerance simple.

7.4 Lemma. Let $V$ be a finite atomistic lattice. Then we have:

(i) Let $a_{1}, a_{2} \in \operatorname{At}(V), a_{1} \neq a_{2}$ and let $d \in V$ be a coatom such that $a_{1} \not \leq d$, $a_{1} \not \leq d$. Then $T\left(\mathbf{0}_{V}, a_{1}\right)=T\left(\mathbf{0}_{V}, a_{2}\right)$.

(ii) If $T\left(\mathbf{0}_{V}, a_{1}\right)=T\left(\mathbf{0}_{V}, a_{2}\right)$ for all $a_{1}, a_{2} \in \operatorname{At}(V)$, then $V$ is tolerancesimple.

Proof. (i): Since $a_{i} \wedge d=0$ and $a_{i} \vee d=1$ for $i=1,2$, we get

$$
T\left(\mathbf{0}_{V}, a_{i}\right)=T\left(d \wedge a_{i}, a_{i}\right)={ }_{(7.3 .1)} T\left(d, d \vee a_{i}\right)=T\left(d, \mathbf{1}_{V}\right),
$$


consequently, $T\left(\mathbf{0}_{V}, a_{1}\right)=T\left(\mathbf{0}_{V}, a_{2}\right)$.

(ii): Since $T\left(\mathbf{0}_{V}, a\right)$ is the same tolerance for each atom $a \in \operatorname{At}(V)$, we will denote it by $\alpha$. We have $\left(\mathbf{0}_{V}, a\right) \in \alpha$ for all $a \in \operatorname{At}(V)$, consequently $\left(\mathbf{0}_{V}, \mathbf{1}_{V}\right)=$ $\left(\mathbf{0}_{V}, \bigvee \operatorname{At}(V)\right) \in \alpha$. From (7.3.2) we get $\alpha=\nabla_{V}$, i.e., $T\left(\mathbf{0}_{V}, a\right)=\nabla_{V}$ for all $a \in \operatorname{At}(V)$. As mentioned above in 7.3, this implies tolerance-simplicity of $V$.

Now, instead of the abstract lattice $V$, we return to the concrete lattice $\mathcal{E}$. Recall that $\mathcal{E}$ is atomistic and $\operatorname{At}(\mathcal{E})=\left\{E_{\varkappa} \mid \varkappa \in \operatorname{Eq}(A)\right\}$ where $E_{\varkappa}=\{\Delta, \varkappa, \nabla\}$ (Theorem 4.1). The least and greatest elements are $\mathbf{0}_{\mathcal{E}}=\{\Delta, \nabla\}$ and $\mathbf{1}_{\mathcal{E}}=$ $\mathrm{Eq}(A)$. As defined in 2.3, $[a, b]$ denotes the equivalence relation (on $A$ ) with one nontrivial block $\{a, b\}$.

7.5 Lemma. Let $\varkappa \in \operatorname{Eq}(A) \backslash\{\Delta, \nabla\}$. Then there exists $(a, b) \in \varkappa, a \neq b$, such that $T\left(\mathbf{0}_{\mathcal{E}}, E_{\varkappa}\right)=T\left(\mathbf{0}_{\mathcal{E}}, E_{[a, b]}\right)$.

Proof. Since $\varkappa$ is nontrivial there exist distinct elements $a, b, c \in A$ such that $(a, b) \in \varkappa$ but $(a, c) \notin \varkappa$. Clearly $(a, b) \in[a, b] \subseteq \varkappa$. Define $f \in A^{A}$ via $f x:=c$ if $x=a$, and $f x=x$ otherwise. Then $f=f^{2}$ is of type (I) and therefore $\operatorname{Con}(A, f)$ is a coatom in $\mathcal{E}$ (cf. Theorem 4.3). Since $(f a, f b)=(c, b)$, the function $f$ preserves neither $\varkappa$ nor $[a, b]$. Hence $\varkappa,[a, b] \notin \operatorname{Con}(A, f)$, consequently the atoms $E_{\varkappa}, E_{[a, b]}$ are not contained in $\operatorname{Con}(A, f)$ and therefore, by applying Lemma 7.4(i), we obtain $T\left(\mathbf{0}_{\mathcal{E}}, E_{\varkappa}\right)=T\left(\mathbf{0}_{\mathcal{E}}, E_{[a, b]}\right)$.

7.6 Theorem. For $|A| \geq 4$, the lattice $\mathcal{E}$ is tolerance simple.

Proof. In view of Lemma 7.4(ii) it is sufficient to show

$$
T\left(\mathbf{0}_{\mathcal{E}}, E_{\varkappa_{1}}\right)=T\left(\mathbf{0}_{\mathcal{E}}, E_{\varkappa_{2}}\right) \text { for all atoms } E_{\varkappa_{1}}, E_{\varkappa_{2}},
$$

i.e., for all $\varkappa_{1}, \varkappa_{2} \in \operatorname{Eq}(A) \backslash\{\Delta, \nabla\}$. Due to Lemma 7.5 we even may restrict to equivalence relations of the form $\varkappa_{1}=\left[a_{1}, b_{1}\right], \varkappa_{2}=\left[a_{2}, b_{2}\right]$ for $\left(a_{1}, b_{1}\right),\left(a_{2}, b_{2}\right) \in$ $A^{2} \backslash \Delta$. If $\left\{a_{1}, b_{1}\right\}=\left\{a_{2}, b_{2}\right\}$, then $E_{\left[a_{1}, b_{1}\right]}=E_{\left[a_{2}, b_{2}\right]}$ and we are done. Hence, w.l.o.g, we can restrict to the following two cases:

Case (a): $a_{1}=a_{2}$ and $b_{1} \neq b_{2}$.

Since $|A| \geq 4$ there exists an element $c \in A \backslash\left\{a_{1}, b_{1}, b_{2}\right\}$. Define $f \in A^{A}$ by $f x=c$ if $x \in\left\{b_{1}, b_{2}\right\}$ and $f x=x$ otherwise. Then $f$ is of type (I) and $\operatorname{Con}(A, f)$ is a coatom (cf. Theorem 4.3). From the definition immediately follows that $f$ does not preserve neither $\varkappa_{1}=\left[a_{1}, b_{1}\right]$ nor $\varkappa_{2}=\left[a_{2}, b_{2}\right]$. Consequently $E_{\varkappa_{1}} \nsubseteq$ $\operatorname{Con}(A, f), E_{\varkappa_{2}} \not \subseteq \operatorname{Con}(A, f)$ and from Lemma 7.4(i) we conclude $T\left(\mathbf{0}_{\mathcal{E}}, E_{\varkappa_{1}}\right)=$ $T\left(\mathbf{0}_{\mathcal{E}}, E_{\varkappa_{2}}\right)$.

Case (b): $\left\{a_{1}, b_{1}\right\} \cap\left\{a_{2}, b_{2}\right\}=\emptyset$.

Consider the permutation $f:=\left(a_{1} a_{2}\right)\left(b_{1} b_{2}\right)$ (with two cycles of length 2). By Theorem 4.3, Con $(A, f)$ is a coatom (type (III)) and we have $f \not \triangleright \varkappa_{1}, f \not \varkappa_{2}$ and as in case (a) above we get $T\left(\mathbf{0}_{\mathcal{E}}, E_{\varkappa_{1}}\right)=T\left(\mathbf{0}_{\mathcal{E}}, E_{\varkappa_{2}}\right)$.

The investigation of lattice properties around modularity shows that such properties cannot be expected for $\mathcal{E}$ : 
7.7 Proposition. For $|A| \geq 4$, the lattice $\mathcal{E}$ has none of the following properties: 0-modular, 1-modular, lower semimodular, upper semimodular.

Proof. If $A$ has at least 4 elements, say $0,1,2,3$, then consider the nontrivial equivalence relations $\varkappa_{1}=[0,1,2], \varkappa_{2}=[0,1][2,3], \varkappa_{0}=\varkappa_{1} \cap \varkappa_{2}=[0,1]$ and the function $f \in A^{A}$ defined by $f x=3$ if $x=0$, and $f x=x$ otherwise.

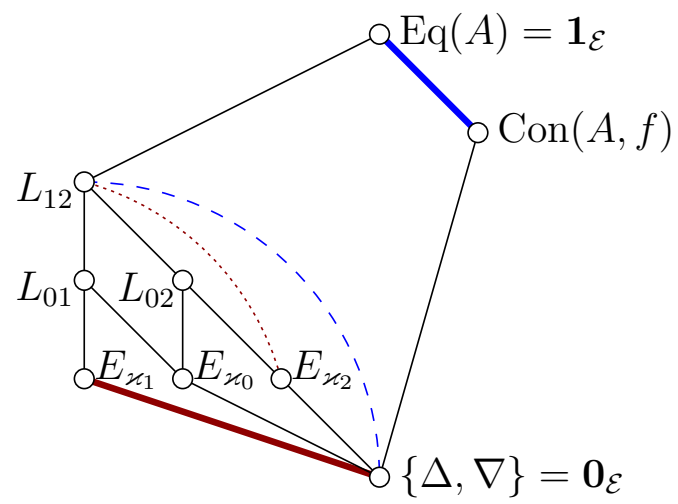

Figure 8: The sublattice of $\mathcal{E}$ used in the proof of Proposition 7.7

We get the sublattice as shown in Figure 8, e.g., $\operatorname{Con}(A, f)$ is a coatom by Theorem 4.3 and it is easy to check that for $L_{i j}:=E_{\varkappa_{i}} \vee E_{\varkappa_{j}}(0 \leq i<j \leq 2)$ we have $L_{12}=\left\{\Delta, \varkappa_{0}, \varkappa_{1}, \varkappa_{2}, \nabla\right\}$ and $L_{0 j}=\left\{\Delta, \varkappa_{0}, \varkappa_{j}, \nabla\right\}(j=1,2)$. Note that $f$ does not preserve neither $\varkappa_{0}$ nor $\varkappa_{1}$ nor $\varkappa_{2}$.

Obviously $\left\{\mathbf{0}_{\mathcal{E}}, E_{\varkappa_{1}}, L_{12}, \operatorname{Con}(A, f), \mathbf{1}_{\mathcal{E}}\right\}$ is a sublattice isomorphic to $N_{5}$. Thus $\mathcal{E}$ is neither 0- nor 1-modular. Further, $\operatorname{Con}(A, f) \prec \mathbf{1}_{\mathcal{E}}$ (blue line) but the meet with $L_{12}$ (dashed blue line) is not a covering; likewise $\mathbf{0}_{\mathcal{E}} \prec E_{\varkappa_{1}}$ (red line) but the join with $E_{\varkappa_{2}}$ (dotted red line) is not a covering. Hence $\mathcal{E}$ is neither lower nor upper semimodular.

7.8 Remark. For $|A|=3$, Theorem 7.6 and Proposition 7.7 do not remain valid. In this case, $\mathcal{E}$ is the lattice of all subsets of $\left\{\Delta, \nabla, \theta_{0}, \theta_{1}, \theta_{2}\right\}$ containing $\Delta$, $\nabla$, where $\theta_{i}, i \in\{0,1,2\}$, are the nontrivial equivalence relation on $A$. Thus it is a Boolean lattice with 8 elements. Therefore it is modular and it is not tolerance-simple (it is even not congruence-simple).

\section{Acknowledgment}

The authors thank S. Reichard for some hints and computations which led to an improved version of Proposition 7.1.

We dedicate our paper to the memory of E. Tamás Schmidt who passed away in 2016. The final version will appear in a special issue of Algebra UnIVERSALIS. Each of the authors is grateful to have known Tamás and to profit from numerous inspiring and friendly discussions with this outstanding mathematician. In particular, the third author is indepted to Tamás as $\mathrm{PhD}$ supervisor 
Jakubíková-Studenovská/Pöschel/Radeleczki, Lattice of congruence lattices 22

introducing him to contemporary lattice theoretical investigations and giving constructive hints concerning the finite representation problem.

\section{References}

[GräS63] G. Grätzer AND E. T. SChMIDT, Characterizations of congruence lattices of abstract algebras. Acta Sci. Math. (Szeged) 24, (1963), 34-59.

[Jak09] D. JakubíkovÁ-Studenovská, Lattice of quasiorders of monounary algebras. Miskolc Mathem. Notes 10(1), (2009), 41-48.

[JakP09] D. Jakubíková-Studenovská And J. Pócs, Monounary Algebras. P.J. Šafárik Univ. Košice, 2009.

[JakPR11] D. Jakubíková-Studenovská, R. Pöschel, And S. Radeleczki, The lattice of compatible quasiorders of acyclic monounary algebras. Order 28(3), (2011), 481-497, (online publication DOI 10.1007/s11083-010-9186-9).

[JakPR13] D. Jakubíková-Studenovská, R. Pöschel, and S. Radeleczki, Irreducible quasiorders of monounary algebras. J. Aust. Math. Soc. 93(3), (2013), 259-276, (online publication DOI 10.1017/S1446788712000328).

[JakPR16] D. Jakubíková-StudenovskÁ, R. Pöschel, And S. Radeleczki, The lattice of quasiorder lattices of algebras on a finite set. Algebra Universalis 75(2), (2016), $197-220$.

[Jan94] M.F. Janowitz, Tolerances, interval orders, and semiorders. Czechoslovak Math. J. 44(119)(1), (1994), 21-38.

[JanR15] M. F. Janowitz And S. Radeleczki, Aggregation on a finite lattice. Order pp. 1-18, online publication DOI 10.1007/s11083-015-9373-9.

[Kin79] M. Kindermann, Über die Äquivalenz von Ordnungspolynomvollständigkeit und Toleranzeinfachheit endlicher Verbände. In: Contributions to general algebra (Proc. Klagenfurt Conf., Klagenfurt, 1978), Heyn, Klagenfurt, 1979, pp. 145-149.

[Pös04] R. Pöschel, Galois connections for operations and relations. In: K. Denecke, M. ERnÉ, AND S.L. Wismath (Eds.), Galois connections and applications, vol. 565 of Mathematics and its Applications, Kluwer Academic Publishers, Dordrecht, 2004, pp. 231-258.

[PösK79] R. Pöschel And L.A. Kalužnin, Funktionen- und Relationenalgebren. Deutscher Verlag der Wissenschaften, Berlin, 1979, Birkhäuser Verlag Basel, Math. Reihe Bd. 67, 1979.

[PösR08] R. PÖSChel AND S. RADELECZKI, Endomorphisms of quasiorders and related lattices. In: G. Dorfer, G. Eigenthaler, H. Kautschitsch, W. More, and W.B. Müller (Eds.), Contributions to General Algebra 18, Verlag Heyn GmbH \& Co KG, 2008, pp. 113-128, (Proceedings of the Klagenfurt Conference 2007 (AAA73+CYA22), Febr. 2007).

[RadS05] S. RADELECZKI AND D. SChWEIGERT, Notes on locally order-polynomially complete lattices. Algebra Universalis 53, (2005), 397-399.

[Wer76] H. WERNER, Which partition lattices are congruence lattices? In: Lattice theory (Proc. Colloq., Szeged, 1974), North-Holland, Amsterdam, 1976, pp. 433-453. Colloq. Math. Soc. János Bolyai, Vol. 14.

[Zád86] L. ZÁdoRI, Generation of finite partition lattices. In: Lectures in universal algebra (Szeged, 1983), vol. 43 of Colloq. Math. Soc. János Bolyai, North-Holland, Amsterdam, 1986, pp. 573-586. 